\title{
Preliminary small mammal taphonomy of FLK NW level 20 (Olduvai Gorge, Tanzania)
}

\author{
Saleta Arcos ${ }^{\mathrm{a}}$, Paloma Sevilla ${ }^{\mathrm{a}}$, Yolanda Fernández-Jalvo ${ }^{\mathrm{b}, *}$ \\ a Departamento de Paleontologia, Facultad de Geología (UCM) e Instituto de Geología Económica (CSIC), C/José Antonio Novais, 2, 28040 Madrid, Spain \\ b Museo Nacional de Ciencias Naturales (CSIC), José Gutiérrez Abascal, 2, 28006 Madrid, Spain
}

Keywords:

Olduvai Bed-I

Small mammals

Taphonomy

Palaeoenvironment

\begin{abstract}
A B S T R A C T
The Bed-I series of Olduvai Gorge (Tanzania) is a reference site in human evolution, having yielded the holotypes of Paranthropus boisei and Homo habilis, together with manufactured artefacts and abundant large and micro-fauna. Excavations in Olduvai Gorge have been recently resumed, with new aims and new results. This paper presents the results of the taphonomic analysis carried out on a fossil small-mammal assemblage recovered from FLK NW level 20, a layer overlying Tuff $C$, dated from $1.84 \mathrm{Ma}$. The analysis provides good evidence of a category 1 predator, most likely a barn owl, as the predator of the bone assemblage. Trampling and sediment compression might influence postdepositional breakage of the bones. This study is especially relevant since previous taphonomic analyses carried out at levels above and below this sample led to inconclusive results due to a low number of fossils (Fernández-Jalvo et al., 1998). The new sample provides new information to reconstruct the paleoenvironmental context in which early hominins inhabited.
\end{abstract}

\section{Introduction}

The analysis of the modifications that taphonomic processes have produced on a fossil assemblage provides information about the past paleobiological and geological agents that might influence species representation in the assemblage. A taphonomic analysis of the microfauna assemblage in a fossil locality is of particular importance in any study involving interpretations based on this fauna, particularly when used as tools for climatic and paleoenvironmental reconstructions (Glue, 1971; Andrews and Evans, 1983; Denys, 1985, 1986; Andrews, 1990; Fernández-Jalvo, 1995; Fernández-Jalvo et al., 1998). Otherwise, inaccurate or even wrong interpretations may derive from the study of the fossil assemblage.

The formation of a fossil vertebrate assemblage follows a series of different taphonomic processes, modifying the carcass from death to recovering as a fossil (Fernández-López, 1999). This sequence of processes may reduce biological information, but in turn adds information involving past environments and fossilization (FernándezLópez, 1981, 1991; Behrensmeyer and Kidwell, 1985). Predation is one of the most common agents that produce small-mammal assemblages (Fernández-Jalvo, 1995; Williams, 2001; Matthews et al., 2005; Gómez, 2005; Montalvo et al., 2008; Stoetzel et al., in press). The distinction of this activity is mainly based on digestion damage, breakage and anatomical element representation (Dodson and Wexlar, 1979; Andrews, 1990; Denys et al., 1995; Williams, 2001). Andrews (1990) could establish a systematic methodology to recognize the

\footnotetext{
* Corresponding author. Fax: + 34915645078.

E-mail address: yfj@mncn.csic.es (Y. Fernández-Jalvo).
}

predator according to traits on the small mammal bones related to the predator's way of ingestion and digestion.

Early dispersion caused by the wind and water prevents a long period for exposure of small mammal bones on the ground (Voorhies, 1969; Dodson, 1973; Wolff, 1973), which, on the other hand, cause bone selection (by size and shape), and breakage and polishing of bone edges depending on the grain size of the sediment (Andrews, 1990). Smallmammal assemblages resulting from predation are protected from weathering by the actual pellet or scat sucture (Andrews, 1990). When pellets and scats disintegrate, bones are exposed to weathering, and the resulting damage is characterized by superficial cracking, splitting and exfoliation until these fall apart (Behrensmeyer, 1978; Andrews, 1990). Trampling can also be responsible for bone dispersal and burial. Trampling occurring in predators dens or nests may lead to serious breakage of small mammal bones, with the breaking of skulls, jaws and long bones of the postcranial skeleton (proximal femora and distal humeri), the reduction in the number of jaws, the loss of teeth in jaws, but no loss or breakage of small elements of postcranial skeleton (Andrews, 1990; Williams, 2001). Once buried, bones may suffer alterations caused by different agents related to properties of the soil or sediment in which they are embedded. These alterations include corrosion caused by soil alkalinity or acidity, attack by microorganisms, superficial damage caused by plant roots and re-mineralization of the bones. Mechanical alterations such as breakage due to sediment compression and compaction may also take place. Occasionally, erosion of sediments containing already buried vertebrate remains can take place, exposing the bones again to some of the previously mentioned agents (weathering, transport, abrasion and trampling). The changes caused by these alterations on already fossilised bones are different to 
those on fresh bones, enabling to recognize reworked bones. Finally, during the process of excavation and the screening of the samples, fossil assemblages may suffer modifications such as breakage or size sorting.

In this paper we describe the fossil small-mammal assemblage from FLK NW level 20 of Bed-I, Olduvai Gorge (Tanzania) from a taphonomic point of view. Especially relevant is that excavations at Olduvai Bed-I have been recently resumed, providing new material and information to compare with previous materials obtained in the 1960's excavations. Bed-I FLK NW level 20 fossil assemblage comes from the new locality FLK NW discovered in 2008, about $100 \mathrm{~m}$ from the locality known as FLK-Zinj. Bed-I is especially important to human evolution as it yielded fossils of Australopithecus and Homo habilis. These fossils were accompanied by other contemporaneous assemblages that provide relevant information about the environment and habitat in which these early hominin species dwelt, and about possible climatic changes during this period.

The FLK sites of Bed-I that contained most archaeological and nearly all of the palaeontological remains were the fossiliferous localities of FLK NN and FLK N. Previous studies during the 1970 s proposed a strong climatic change between the middle (FLK NN) and the top (FLK N) of Bed-I, mainly based on the small-mammal faunal content, which showed a change in the proportions of murines vs. gerbillines. A previous study on the small mammal taphonomy of Bed-I of Olduvai (Fernández-Jalvo et al., 1998) was based on the collection recovered from the old excavations by M.D. Leakey. This study showed evidence of predation in all units of Bed-I. Different predator behaviours could explain some of the variability observed in the small-mammal species composition between the middle and the top of Bed-I. Within the Formation of Bed-I of Olduvai Gorge, Fernández-Jalvo et al. (1998) distinguished three stages according to paleoecological characterization after a detailed taxonomic and taphonomic study of the microfaunal content. The middle Bed-I (FIK NN) showed a very rich dense woodland environment, richer than any of the present-day savanna biomes in Africa. The environment was similar, or gradually changing to less rich woodland in upper Bed-I (FLK N6 5-4) and a trend towards more openand seasonal woodlands atthetop of the series (FLK N 3-2-1), comparable to conditions occurring today in tropical East Africa.

This small-mammal collection, however, did not include the assemblage of FLK NW level 20, now obtained from recent excavations. This fossil assemblage is directly overlying Tuff $C$ (yielding an age of $1.839 \pm 0.005 \mathrm{Ma}$ by ${ }^{40} \mathrm{Ar} /{ }^{39} \mathrm{Ar}$, according to Blumenschine et al., 2003). The closest fossil assemblages to this stratigraphic layer studied in 1998 were FLK-Zinj (below Tuff C) and FLK N6 (above TuffD). Both of them were particularly poor and contained evidence of taphonomical mixtures previous to final burial. Thus, none of them could provide conclusive taphonomic results. The taphonomic study of level FLK NW level 20 aims to fill the gap between Tuff $C$ and Tuff $D$ that was not previously recorded. The identification of the predator provides information about the possibility of bias in the small-mammal assemblage, necessary before any paleoenvironmental interpretation is given. Moreover, the taphonomic traits of FLK NW level 20 may provide criteria to correlate this sample with other units at Bed-I.

\section{Materials and methods}

The material described in this paper was excavated by Dr. Dominguez-Rodrigo and his team in the 2008 season. It was sieved with a $2-\mathrm{mm}$ mesh at the field camp site (smaller screens require the use of water, scarce in field), obtaining a relatively rich sample of small mammal remains. Though the detailed taxonomic study is still to be done, evidence of a dominance of rodent remains is evident at first sight, mainly murine and gerbil species; occasional insectivore remains were also identified in the assemblage. This predominance of rodents, however, must not be considered as conclusive, since we are aware that the use of $2-\mathrm{mm}$ mesh leads to the loss of the smaller remains such as insectivore elements, as well as molars or fragments of other taxonomic groups.

The taphonomic study of the FLK NW level 20 material was carried out following the methodology proposed by Andrews (1990). Apart from the analysis of the material in search of evidences of digestion and the patterns leading to the identification of the possible predator, other observations were considered in order to establish the whole of the events that have caused the modifications observed in this fossil assemblage. Thus, the breakage of the bones, their relative abundances, indices of postcranial/cranial and surface modifications were used to identify processes such as weathering, transport, trampling, soil corrosion, sediment compression or modifications caused during the excavation of the material.

In order to identify surface modifications, the whole collection was observed at variable magnification through a binocular microscope (20-40x) and observations have been backed using an environmental scanning electron microscope (Quanta 200) hosted at the Museo Nacional de Ciencias Naturales of Madrid.

The following indices and calculations were performed to characterise the taphonomy of the FLK NW level 20 small-mammal assemblage:

1. Number of Identified Specimens (NISP, see Lyman, 1994) present in the sample, and Relative Abundances of each element and Minimum Number of Individuals (MNI) (Table 1).

2. The proportion of the different elements in the assemblage (Table 2) has been calculated by means of four different indices (see Andrews, 1990):

a - postcranial remains vs. cranial remains, calculated comparing the number of humeri plus femora to mandibles plus maxillae;

b - the abundance of five postcranial elements (humerus, radius, ulna, femur and tibia) compared to the abundance of mandibles, maxillae and isolated molars;

c - the loss of distal limb elements, as shown by an index comparing the number of tibiae and radii with the number of femora and humeri in the sample;

d - percentage of isolated teeth, calculated from the number of teeth (molars and incisors) in the sample (given in Table 1) compared to the number of empty alveolar spaces in the jaws from which teeth from the sample must have come. Values over $100 \%$ were indicating a higher loss of jaws compared to teeth loss and vice versa; this index was calculated independently for incisors and for molars.

3. The degree of postcranial breakage in the assemblage was inferred comparing for each skeletal element, the number of complete

Table 1

Small-mammal skeletal elements identified in the FLK NW level 20 (Bed-I) sample.

\begin{tabular}{lrr}
\hline Skeletal elements & NISP & צ. F \\
\hline Mandible & 161 & 40.7 \\
Maxilla & 68 & 17.2 \\
Incisor & 316 & 39.9 \\
Molar & 108 & 4.5 \\
Femur & 272 & 68.7 \\
Tibia & 395 & $\mathbf{9 9 . 7}$ \\
Pelvis & 218 & 55.1 \\
Calcaneus & 73 & 18.4 \\
Astragalus & 33 & 8.3 \\
Humerus & 186 & 47.0 \\
Radius & 55 & 13.9 \\
Ulna & 76 & 19.2 \\
Scapula & 40 & 10.1 \\
Vertebra & 595 & 8.3 \\
Metapodial & 257 & 6.5 \\
Phalanges & 77 & 0.7 \\
MNI & 198 & \\
\hline
\end{tabular}


Table 2

Indices of craneal/postcrania and indices of isolated teeth according to Andrews (1990) in FLK NW level 20 (Bed-I) sample.

\begin{tabular}{lc}
\hline Femur + humerus/mandible + maxilla & 200 \\
Postcrania/crania & 467.2 \\
Tibia + radius/femur + humerus & 98.3 \\
Number of isolated molars (sample) & 108 \\
Number of in situ molars & 261 \\
Number of empty alveolar molars in maxillae and mandibles & 426 \\
\% lsolated molars & $25.35 \%$ \\
Number of isolated incisors & 316 \\
Number of in situ incisors & 72 \\
Number of empty alveolar incisors in maxillaeand mandibles & 157 \\
$\%$ Isolated incisors & $201.2 \%$ \\
\hline
\end{tabular}

bones, against the number of proximal, shaft and distal fragments (Table 3).

4. Skull breakage was calculated comparing the proportion of unbroken skulls preserved in the sample with the proportion of maxillae retaining the zygomatic process. The percentage of maxillar molar loss and maxillar incisor loss is the number of empty alveolar spaces in the maxillae compared with total number of teeth that should be present (Table 4).

5. The degree of mandible breakage was expressed by the proportion of complete hemi-mandibles (no distinction was made between left and right), the proportion of mandibular bodies with inferior border broken. The percentage of molars and incisors loss is obtained from the number of mandibles without molars and incisors compared with total number of teeth that should be present (Table 4). A new breakage category, consisting of mandibles broken at the diasteme was included, and considered as a higher degree of breakage, than the categories previously described by Andrews (1990) due to predation, and possibly caused by trampling.

6. Recent breakage in large bones of postcranial skeleton during collecting and sieving was identified and quantified (humerus, femur and tibia, Table 3). This type of breakage is characterized by broken edges with a different color and texture than the remaining fossil surface. Recent breakage edges are not covered with sediment and some recent broken bones have remains of tubular sediment casts preserved at the interior of the marrow cavity of long bones.

Table 3

Breakage in postcranial and reont breaknge in FLK NW level 20 (Bed-1) sample.

\begin{tabular}{lcc}
\hline Postcranial elements & NISP & \\
& & \\
\hline Humerus & 30 & \\
Complete & 38 & 16.1 \\
Proximal & 22 & 20.4 \\
Shaft & 96 & 11.8 \\
Distal & 43.83 & 51.6 \\
Recent/Old breakage & & 56.16 \\
Ulia & 1 & \\
Complete & 75 & 1.32 \\
Proximal & 0 & 98.7 \\
Shaft & 0 & 0 \\
Distal & & 0 \\
Femur & 30 & 11.02 \\
Complete & 169 & 62.1 \\
Proximal & 26 & 9.5 \\
Shaft & 47 & 17.2 \\
Distal & 52.65 & 47.34 \\
Recent/Old breakage & & \\
Tibia & 6 & 1.51 \\
Complete & 96 & 24.3 \\
Proximal & 150 & 37.9 \\
Shaft & 143 & 36.2 \\
Distal & 46.13 & 53.86 \\
Recent/Old breakage & & \\
\hline
\end{tabular}

Table 4

Breakage of mandibles and maxillae in FLK NW level 20 (Bed-I) sample.

\begin{tabular}{lc}
\hline Breakage of skulls & \\
\% Complete skulls & 0 \\
\% Maxillae with zigomatic & 8 \\
\% Max. molar loss & 12.8 \\
\% Max. incisor loss & 100 \\
Breakage of mandible & \\
\% Complete mandibles & 0.6 \\
\% Broken ascending ramus & 16 \\
\% Asoending ramus missing & 30.6 \\
\% Inferiorborder broken & 24.0 \\
\% Broken diasteme & 28.6 \\
\% Mand. molar loss & 15.32 \\
\% Mand. incisor loss & 38 \\
\hline
\end{tabular}

7. Bone surface was studied in order to identify any evidence of corrosion, root marks. Breakage by pressure is recognized as bones showing local depressions surrounded by collapsed fragments, forming clusters cemented by the carbonatic sediment of the site (Fig. 1). These fragments might fall apart during screening. Postdepositional damages have been described as presence/ absence but not quantified or displayed in a table.

8. Digestion processes were analysed both in the postcranial elements as in the dental elements. The degree of postcranial digestion was calculated considering evidence in the head of femora and the distal end of humeri. The number of bones with any of these signs of digestion was compared to the total number of femora and humeri. To evaluate digestion processes in the dental remains, molars and incisors were analysed separately and distinguished between the teeth in situ in their alveoli and the isolated teeth in the sample. Table 5 shows the number of teeth with signs of digestion compared to the total number of teeth. Within the digested teeth, the pattern and degree of digestion were considered and related to the classifications found in Andrews (1990), Fernández-Jalvo and Andrews (1992) and Williams (2001). According to these authors, five different categories of digestion exist depending on the degree and percentage of digested elements in the sample, together with type and intensity of bone breakage and the loss of anatomical elements (Andrews (1990)). Each category includes specific predators: nocturnal owls in categories 1-3, and diurnal birds of prey and mammalian predators in categories 4 and 5 .

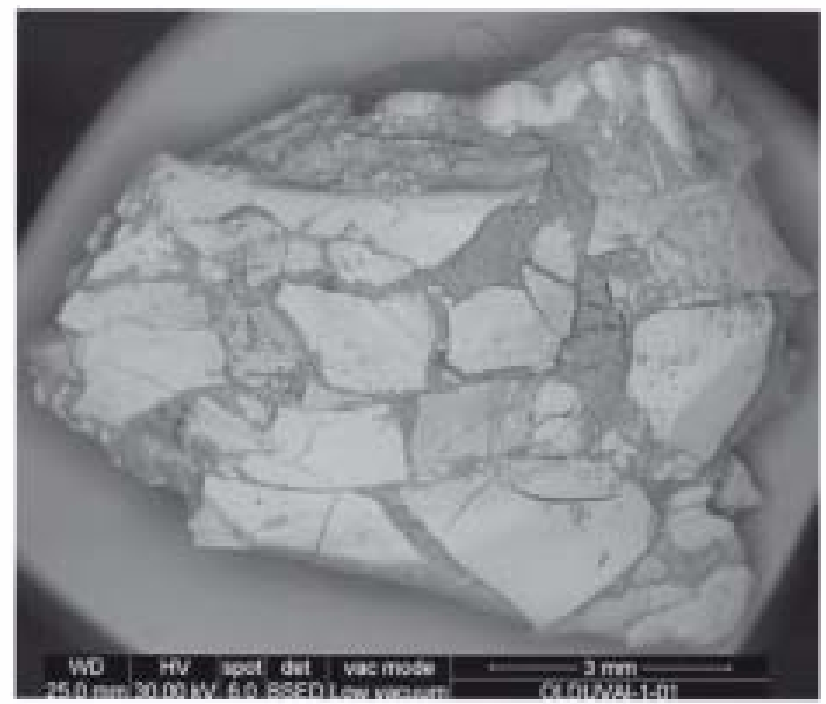

Figure 1. SEM micrograph of surface compression surrounded by collapsed fragments and cemented by the carbonatic sediment of the site. 
Table 5

Traits of digestion in postcranial (proximal femur and distal humerus) and teeth (incisors and molars) in FLK NW level 20 (Bed-I) sample.

\begin{tabular}{ll}
\hline Digested incisors & \\
$\%$ Tip & 6.3 \\
$\%$ Surface & 1.0 \\
$\%$ Light & 7.3 \\
$\%$ Moderate & 0 \\
$\%$ Heavy & 0 \\
$\%$ Extreme & 0 \\
Molars digested & \\
$\%$ Light & 0 \\
$\%$ Moderate & 0 \\
$\%$ Heavy & 0 \\
$\%$ Extreme & 0 \\
Digestion of postcrania & \\
$\%$ Proximal femora digested & 0 \\
$\%$ Distal humeri digested & 0 \\
\hline
\end{tabular}

\section{Results}

Table 1 shows the different skeletal elements represented in sample FLK NW level 20, with a total number of identified specimens (NISP) of 2930, and a MNI of 198 based on the most abundant anatomical element recorded in the sample. Tibia, followed by femora, pelves, mandibles and incisors are the most abundant skeletal elements. The smallest bones, such as phalanges, metapodials or tarsals, together with isolated molars are scarce when compared to the relative abundance of these elements that should be expected for 198 individuals. The wide mesh $(2 \mathrm{~mm})$ used for sieving is a feasible cause of this loss. This has not strongly affected long bones, flat bones (pelves) or incisors that, because of their shape, are more easily retained in the mesh.

Indices relating postcranial and cranial elements indicate a high predominance of postcranial compared to cranial elements, while distal limb bones (tibia, radii,) have similar abundances to proximal limb bones (femora and humeri) (Table 2). The number of isolated incisors compared to the number of empty alveoli in mandibles and maxillae suggest an important rate of destruction of jaws (Table 2). The relative abundance of maxillae (17.2\%) is especially low relative to the expected number that should have been recovered, indicating that destruction of jaws is mainly referred to maxillae. This is also indicated by the high percentage of incisor loss from maxillae (100\%) compared to the loss observed in the mandibles (38\%) (Table 4).

With regard to digestion, $7.3 \%$ of the incisors show evidence of corrosion by gastric juices (Fig. 2), slight in all cases, and in 6.3\% restricted to the tip (Table 5 ). No evidence of digestion was observed either on molars or on postcranial elements, indicating a very low rate of digestion. Breakage before digestion is completely absent.

Taphonomic breakage was analysed considering both the degree and the type of breakage and was observed both in the long bones (Table 3) and in jaws (Table 4). The result was that, as a whole, FLK NW level 20 assemblage showed a high degree of breakage. Among the cranial remains, no complete maxilla is preserved and only $8 \%$ of the remaining material preserves the zygomatic process (Table 4). No maxillary fragment preserved the incisors in situ, although $12.8 \%$ of molars were missing from the alveoli. Fractured jaws are dominant in the sample, with only $0.6 \%$ of them preserved complete. The percentages of incisor and molar loss are $38 \%$ and $15.3 \%$, respectively. Mandibles are frequently broken at the level of the diasteme preserving the incisor socket (28.6\%), indicating an intensive breakage. In spite of the high rate of breakage observed in mandibles and maxillae, a number of long bones in the sample are preserved unbroken, particularly the most robust (femora and humeri) (16\% and $11 \%$ respectively, Table 3 ). Similarly, the number of molars retained in situ is relatively high considering the high destruction observed in jaws.

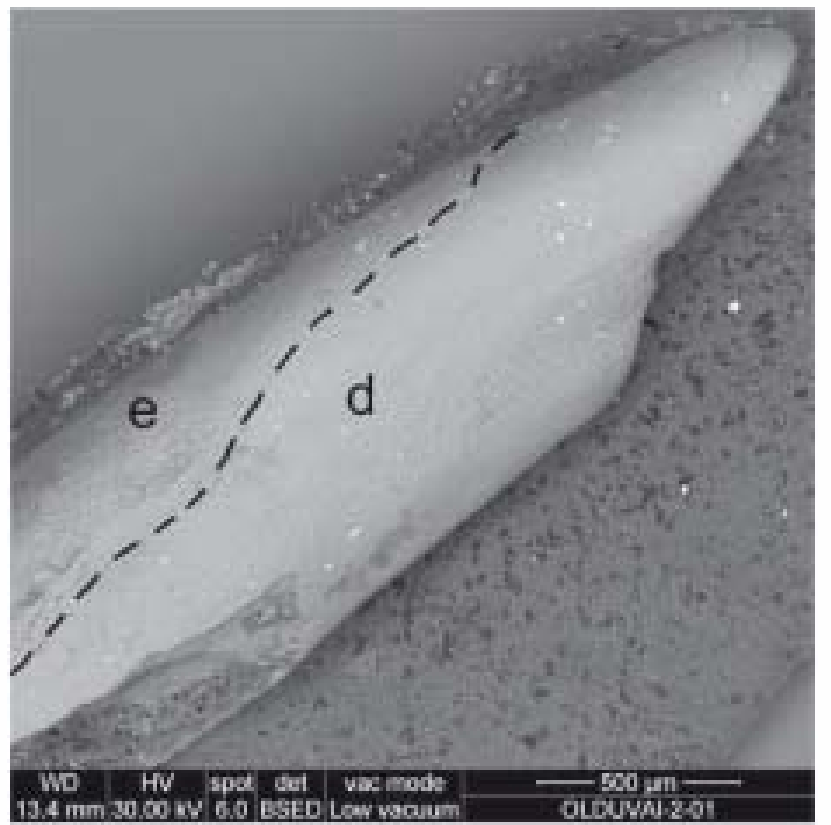

Figure 2. SEM micrograph of a small mammal light digestion concentrated on the tip. Digestion affects the enamel producing a pitted surface and eating away the enamel at the edge of the incisor tip.

Recent breakage is observed in approximately a 50\% of the long bones in the sample (femora, humeri and tibiae) (Table 3). Breakage during collection and sieving was also considered. Nevertheless, these apparently high percentages are lower than those referred in previous studies (Fernández-Jalvo et al, 1998), where the values of recent breakage observed in the sample ranged from a $66 \%$ to a $96 \%$.

Breakage related to pressure has been observed in the fossil assemblage of FLK NW level 20 (Fig. 1). No signs of transport before burial nor mixtures were distinguished, and damage by rootmarks or weathering were not identified. Soil corrosion has been observed as a superficial desquamation at FLK NW level 20. This is especially characteristic of Bed-I fossil assemblages, described by FernándezJalvo et al. (1998) as a superficial exfoliation typical of late stages of weathering, without the previous cracking characteristic of early stages of weathering. This characteristic exfoliation was referred as "desquamation" in order to distinguish it from weathering. Desquamation was experimentally obtained from highly alkaline solutions $(\mathrm{pH} \approx 14)$, and observed in fossil assemblages from cave taphosystems and at Olduvai Bed-I. Desquamation has been thus linked to alkaline soil corrosion.

\section{Discussion: interpretation of taphonomic processes}

The presence of incisors with some evidence of digestion suggests the participation of a predator in the formation of the assemblage. Breakage before digestion, as result of tearing apart or chewing the prey during ingestion, is completely absent. All this suggest a nocturnal predator. The percentage of remains modified by digestion is very low, occurring at a light degree only on incisors and predominantly on the tip, indicating that incisors were retained in the jaws during digestion. The relatively high rate of molars preserved in situ and absence of digestion on femora or humeri suggest the involvement of a category 1 predator in FLK NW level 20. The very low rate of digestion observed in this sample and indication of low destruction due to predation, ingestion and digestion of the prey indicates a representative at the lowest end of Category 1. This category includes species such as barn owls (Tyto alba), snowy owls (Bubo scandiacus) and great grey owls (Strix nebulosa) following Andrews' (1990) classification. Both the snowy owl and the great grey 
owl are species distributed in higher latitudes and absent in Africa. Barn owls are found in African ecosystems, and the action of this predator was also identified in the study of Fernández-Jalvo et al. (1998) of the FLK NN2 assemblage. Therefore, the most feasible candidate as predator responsible for the fossil assemblage of FLK NW level 20 is the barn owl.

Transport processes as responsible for the patterns of modification in the FLK NW level 20 assemblage were discarded on the basis of the following argument. The values shown in Table 1 suggest lack of element sorting according to the criteria established by Voorhies (1969). In the classifications based on the hydro/aero dynamic features of the different skeletal elements, pelves and femurs belong to categories I/II (in other words, these bones are easily transported), whereas mandibles and tibiae are included in category $V$, which groups the last elements to be removed by transport from an assemblage (Voorhies, 1969; Dodson, 1973; Behrensmeyer, 1975; Korth, 1979). It is easily observed that the numbers representing the different bone elements in the assemblage do not agree with sorting involving transport. No abraded surfaces or rounded edges were observed in the bones. Accordingly, transport is discarded as a possible taphonomic agent influencing the formation of the FLK NW level 20 assemblage.

A high degree of post-predation fracture is shown from the study of breakage of cranial and postcranial elements. Indications of preburial trampling is evidenced by abundant breakage of the more fragile elements, mainly maxillae, compared to more robust postcranial elements (mainly long bones, more frequently found complete in the sample) and mandibles. The pattern that emerges from trampling was described by Andrews (1990) as result of experimental work, marked by the absence of complete skulls, a low number of maxillae, the significant high number of detached teeth, as well as the high proportions of distal humeri and proximal femora. Andrews (1990) noted that trampling of excrements in mammal dens and of pellets in owl and diurnal raptor nests result in the destruction of skulls, jaws and postcranial elements. This pattern of trampling processes agrees well with observations in the FLK NW level 20 material, and possibly took place within the nest since no signs of transport were detected. The relative presence of complete bones would suggest that, even though intensive trampling took place, the original assemblage produced by predators had a low grade of breakage.

Another type of breakage, that caused by pressure (Fig. 1), was observed on fossils from Bed-I by Fernández-Jalvo et al. (1998). This type of breakage, described as "local depressions surrounded by collapsed fragments" was observed to be on a par with trampling processes; in other words, it was present where signs of trampling were frequent. At FLK NW level 20, trampling is also intense and breakage by pressure is also recurrent. However, a certain degree of post-burial breakage when embedded in sediment should also be considered, because these broken and compressed fragments are cemented by carbonatic sediment. This could only have taken place once the bone was already buried. Thus, though trampling could have been the main cause for this particular type of breakage, it probably occurred when the remains were already buried superficially.

Finally, broken edges with different color and texture indicate a more recent breakage. The use of $2-\mathrm{mm}$ screens in the field may be partly responsible for bone breakage in the assemblage, as well as the loss of isolated teeth and smaller bones, including fragments of larger bones from breakage that took place before and after burial.

Comparing results here obtained to those previously described by Fernández-Jalvo et al. (1998), we could observe a high percentage of rounding and polishing that characterizes FLK-Zinj, FLK N6 and FLK N5 assemblages, whereas rounding and polishing is less abundant at the rest of the Bed-I units previously studied and absent at FLK NW level 20 here studied. Breakage by pressure described above was also observed elsewhere at these fossil assemblages: it was abundant at FLK N5 and FLK N6 (immediately above Tuff D and FLK NW level 20) but absent at FLK-Zinj. Trampling was observed to be especially abundant at FLK N6 and 5, but absent at the FLK NN series and FLKZinj. Evidence of postdepositional mixtures were observed at FLK-Zinj and FLK N6, preventing the identification of the predator. Mixtures were absent at other units of Bed-I and at the sample FLK NW level 20. Some bones affected by weathering were present at FLK-Zinj, FLK N5, 4 and 2, but absent at FLK NW level 20, FLK N6, 3 and 1, and FLK NN2 and 3; nevertheless, percentages were invariably low. Root marks were also very infrequent and only slightly evident at FLK N4 and FLK N5. Soil corrosion by alkaline sediments produced superficial desquamation on some fossils at all units of Bed-I, and has been also recognized at the new sample from FLK NW level 20 described in this paper.

\section{Conclusions}

The taphonomic analysis carried out with the material of small mammals from the FLK NW level 20 of Olduvai Bed-I and presented in this study leads to the conclusion that the remains present all the characteristics imposed on a mammal assemblage accumulated by a nocturnal bird of prey, most likely a barn owl (Tyto alba). Our conclusion is based on the fact that signs of digestion in the material are absent or very slight. In spite of a high rate of breakage due to postdepositional (trampling) and post-burial conditions (sediment compaction) or recent breakage, there are signs of low breakage by predation (e.g., complete postcranial robust elements and molars in situ), agreeing with our interpretation concerning the predator. Since barn owls are non-selective (apart from their relative small size and nocturnal preferences) and opportunistic predators that catch their prey in the immediate surroundings (Mikkola, 1983, Cramp, 1985), assemblages originated from their pellets provide a good view of the small-mammal communities in the area. Thus, a paleoecological interpretation for FLK NW level 20 based on the small-mammal assemblage may be considered reliable.

The most relevant postdepositional modifications identified at a previous taphonomic study carried out at Bed-I (Fernández-Jalvo, et al., 1998), more specifically levels below (FLK-Zinj) and above (FLK N6 and 5) have also been observed in the FLK NW level 20 sample. In general, indications of prolonged exposure on the ground surface are not evident at FLK NW level 20. A slightly higher similarity with regard to postdepositional conditions of FLK NW level 20 is observed with FLK N6 and 5, and the presence of desquamation is affecting the fossil bone surfaces all along Bed-I, linked to alkaline sediments. Thus, equivalent conditions for fossilization have been interpreted, making possible the comparison of these assemblages and enabling the use of the resulting differences to identify environmental changes.

\section{Acknowledgments}

We are grateful to Manuel Dominguez-Rodrigo for allowing us to study this fossil assemblage. We are also thankful to reviewers for their comments and suggestions that have greatly improved the paper. English translation of the original text was done by Maya Mukerjee. This analysis has been supported by project CGL200766231 of the Spanish Ministry of Science and Innovation.

\section{References}

Andrews, P., 1990. Owls, caves and fossils. Natural History Museum Publications, London.

Andrews, P., Evans, E.M.N., 1983. Small mammal bone accumulations produced by mammalian carnivores. Paleobiology 9, 289-307.

Behrensmeyer, A.K., 1975. The taphonomy and paleoecology of plio-pleistocene vertebrate assemblages east of Iake Rudolf, Kenya. Bulletin of the Museum of Comparative Zoology 145 (10), 473-574.

Behrensmeyer, A.K., 1978. Taphonomic and ecologic information from bone weather ing. Paleobiology 4, 150-162. 
Behrensmeyer, A.K., Kidwell, S.M., 1985. Taphonomy's contributions to paleobiology. Paleobiology 11 (1), 105-119. Published by: Paleontological Society Stable URL: http://www.jstor.org/stable/2400427.

Blumenschine, R.J., Peters, C.R., Masao, F.T., Clarke, R.J., Deino, A.L., Hay, R.L., Swisher, C.C., Stanistreet, LG., Ashley, G.M., McHenry, L.J., Sikes, N.E., van der Merwe, N.J., Tactikos, J.C., Cushing, A.E., Deocampo, D.M., Njau, J.K., Ebert, J.L, 2003. Late Pliocene Homo and hominid land use from Western Olduvai Gorge, Tanzania. Science 299, 1217-1221.

Cramp, S. (Ed.), 1985. The birds of the western Palearctic, voL 4. Terns to woodpeckers. Oxford University Press, Oxford.

Denys, C., 1985. Nouveaux critères de reconaissance des concentrations de microvértebres d'après l'étude des pelotes de chouettes du Bostwana (Afrique australe): Bulletin du Muséum National d'Histoire Naturelle, Paris, 7, pp. 879-933.

Denys, C., 1986. Le gisement Pliocene de Laetoli (Tanzanie, Afrique de I'Est): analyse taphonomique des assemblages de microvertebres. Paleontographica 194, 69-98.

Denys, C., Fernández-Jalvo, Y., Dauphin, Y., 1995. Experimental taphonomy: preliminary results of the digestion of micromammal bones in laboratory: Comples Rendus de l' Académie des Sciences, Serie Ila, 321, pp. 803-809.

Dodson, P., 1973. The significance of small bones in paleoecological interpretation. Contributions to Geology $12,15-19$.

Dodson, P., Wexlar, D., 1979. Taphonomic investigations of owl pellets. Paleobiology 5 , 275-284.

Fernández-Jalvo, Y., 1995. Small mammal Taphonomy at la Trinchera de Atapuerca (Burgos, Spain). A remarkable example of taphonomic criteria used for stratigraphic correlations and palaeoenvironmental interpretations. Palaeogeography, Palaeoclimatology, Palaeoecology 114, 167-195.

Fernández-Jalvo, Y., Andrews, P., 1992. Small mammal taphonomy of Gran Dolina, Atapuerca (Burgos), Spain. Journal of Archaeological Science 19, 407-428.

Fernández-Jalvo, Y., Denys, C., Andrews, P., Williams, T., Dauphin, Y., Humphreys, L. 1998. Taphonomy and palaeoecology of Olduvai Bed-I (Pleistocene, Tanzania). Journal of human evolution $34,137-172$.
Fernández-Iópez, S., 1981. Laevolución tafonómica (un planteamiento neodarwinista): Boletin de la Real Sociedad Española de Historia Natural (Seccion Geologica), 79, pp. 243-254

Fernández-Iópez, S., 1991. Taphonomic concepts for a theoretical biochronology Revista Española de Paleontología 6, 37-49.

Fernández-Iópez, S., 1999. Tafonomía y fosilización. In: Meléndez, B. (Ed.), Tratado de Paleontología. Consejo Superior de Investigaciones Científicas, Madrid, pp. 51-107.

Glue, D.E., 1971. Avian predator pellet analysis and the mammalogist. Mammal Review $1,53-62$.

Gómez, G., 2005. Analysis of bone modifications of Bubo virginianus' pellets from Argentina. Journal of Taphonomy 3, 1-16.

Korth, W.W., 1979. Taphonomy of microvertebrate fossil assemblages. Annals of the Carnegie Museum 48, 235-285.

Lyman, R.L., 1994. Vertebrate taphonomy. Cambridge University Press, Cambridge

Matthews, T., Denys, C., Parkington, J.E., 2005. The palaeoecology of the micromammals from the late middle Pleistocene site of Hoedjiespunt 1 (Cape Province, South Africa). Journal of Human Evolution 49, 432-451.

Mikkola, H., 1983. Owls of Europe. T. \& A.D. Poyser, Staffordshire.

Montalvo, C.L, Melchor, R.N., Visconti, G., Cerdeño, E., 2008. Vertebrate taphonomy in loess-palaeosol deposits: a case study from the late Miocene of central Argentina. Geobios 41, 133-143.

Stoetzel, E., Marion, L., Nespoulet, R., Abdeljalil El Hajraoui, M., Denys, C., in press. Taphonomy and Palaeoecology of the Late Pleistocene to Middle Holocene Small Mammal succession of El Harhoura 2 cave (Rabat-Témara, Morocco). Journal of Human Evolution.

Voorhies, M.R., 1969. Taphonomy and population dynamics of an earlyPliocene vertebrate fauna, Knox County, Nebraska. Contributions to Geology, Special paper 1, 1-69.

Williams, J.P., 2001. Small mammal deposits in archaeology: a taphonomic investigation of Tyto alba (barn owl) nesting and roosting sites. PhD. Thesis. University of Sheffield.

Wolff, R.G., 1973. Hydrodynamic sorting and ecology of a pleistocene mammalian assemblage form California (USA). Palaeogeography, Palaeoclimatology, Palaeoecology 13, 91-101. 